\title{
Prediction of Ambulatory Status After Hip Fracture Surgery in Patients Over 60 Years Old
}

\author{
Jae Lim Kim, MD, Ji Sun Jung, MD, Sang Jun Kim, MD, PhD \\ Department of Physical and Rehabilitation Medicine, Samsung Medical Center, \\ Sungkyunkwan University School of Medicine, Seoul, Korea
}

\begin{abstract}
Objective To predict ambulatory capacity, 1 month after physical therapy following hip fracture surgery.
Methods A retrospective chart review was carried out. Patients more than 60 years old, who underwent hip fracture surgery and received physical therapies, were selected $(n=548)$. Age, gender, presence of cognitive dysfunction, combined medical diseases, combined fractures, previous history of hip surgery, prefracture ambulatory capacity, days from the fracture to surgery, type of fracture, type of surgery, presence of postoperative complications, days from the surgery to physical therapy, and total admission period, were collected. Prefracture ambulatory capacity and postoperative ambulatory capacity were classified into non-ambulatory status (NA), ambulation with assistive device (AA), and independent-ambulation without any assistive device (IA). Multiplelogistic regression analysis was performed for the prediction of postoperative ambulatory capacity.

Results Age (odds ratio [OR]=0.94 for IA and 0.96 for IA or AA), gender (OR=1.64 for IA and 0.98 for IA or AA), prefracture ambulatory capacity (OR of IA=19.17 for IA; OR of IA=16.72 for IA or AA; OR of AA=1.26 for IA, OR of $\mathrm{AA}=9.46$ for IA or $\mathrm{AA})$, and combined medical disease $(\mathrm{OR}=2.02)$ were found to be the factors related to postoperative ambulatory capacity and the prediction model was set up using these four factors.

Conclusion Using this model, we can predict the ambulatory capacity following hip fracture surgery. Further prospective studies should be constructed to improve postoperative ambulatory capacity.
\end{abstract}

Keywords Hip fractures, Walking, Dependent ambulation, Prognosis, Aged, Surgery

\section{INTRODUCTION}

Patients over 60 years old have a high tendency of falling down, because they have risk factors including muscle weakness, poor vision, hypnotics or anti-depressants, and combined medical diseases [1]. Falls in the elderly are closely connected with hip fractures, because their bones are more fragile due to osteoporosis [2]. According to a previous report [3], the incidence of falling in people aged 65 years or over was $13 \%$, and this falling down pro-

Received September 16, 2015; Accepted December 16, 2015

Corresponding author: Sang Jun Kim

Department of Physical and Rehabilitation Medicine, Samsung Medical Center, Sungkyunkwan University School of Medicine, 81 Irwon-ro, Gangnamgu, Seoul 06351, Korea. Tel: +82-2-3410-6069, Fax: +82-2-3410-0052, E-mail: guitarren.kim@samsung.com

ORCID: Jae Lim Kim (http://orcid.org/0000-0001-8267-2748); Ji Sun Jung (http://orcid.org/0000-0001-8579-5794); Sang Jun Kim (http://orcid. org/0000-0002-0479-7526).

(c) This is an open-access article distributed under the terms of the Creative Commons Attribution Non-Commercial License (http://creativecommons.org/ licenses/by-nc/4.0) which permits unrestricted noncommercial use, distribution, and reproduction in any medium, provided the original work is properly cited. Copyright (c) 2016 by Korean Academy of Rehabilitation Medicine 
gressed into hip fracture in $3.7 \%$ of subjects.

Hip fractures in patients over 60 years old are catastrophic events that often lead to dramatic consequences, such as gait impairment, limitation of daily activities, and death [4]. In patients over 60 years old with hip fracture, early mobilization is an important issue, because the ambulatory status is a predictive factor for 1-year mortality after hip surgery [5]. Therefore, for regaining quality of life and with respect to mortality after hip fracture, recovery of the ambulatory capacity is important.

The ambulatory capacity after hip fracture surgery can be divided into non-ambulatory state, ambulation with assistive device, and ambulation without assistive device. In patients over 60 years old who have gait disorders, single cane or walkers must be utilized as an assistive device according to their balance and weight bearing capacity [6]. A single cane can support approximately one quarter of a person's body weight, while a walker can support approximately half of a person's body weight and improve a patient's balance by increasing the base of support [7].

There have been many studies assessing functional status after hip fracture surgery and prognostic factors of functional status [8-13]. However, none of the studies assess whether a patient after hip fracture surgery could walk without any assistive device, or walk only with the assistance of a device, or could not walk independently. The use of an assistive device, especially a walker, will prevent patients over 60 years old from falling, but it is limited for stepping up and down or walking on uneven ground. In addition, involving the upper extremities for using the device during walking makes it difficult for a person to perform certain activities, such as opening or closing a door. Therefore, a person who can walk without a walker is more satisfied with their quality of life.

After rehabilitation, ambulatory function of patients varied. Some people can walk and some cannot walk independently. Prediction of mobility would be helpful to establish the goal of rehabilitation. If we can predict whether a person can walk without any device, or can only walk with the assistance of an ambulatory device, or cannot walk after hip fracture surgery, this information will be useful for patients over 60 years old undergoing hip fracture surgery to perform their daily activities after surgery. Therefore, the aim of this study was to predict the ambulatory capacity after physical therapy following hip fracture surgery.

\section{MATERIALS AND METHODS}

\section{Participants}

This was a retrospective study conducted by medical chart review. A total of 564 patients of more than 60 years old, who were admitted for hip fracture to the Department of Orthopedic Surgery in Samsung Medical Center, underwent hip fracture surgery, received physical therapies, and had medical records concerning ambulatory capacities before falling down and 1 month after surgery from January 2006 to June 2013, were selected. Among them, 6 patients in whom the time from onset to surgery was more than 2 months were excluded, because they did not receive surgery for achieving ambulation. In 10 patients who received surgery more than two times during the study period, their initial data was included in our study to prevent overlap. Finally, a total of 548 patients were included in this study.

Physical therapy was initiated when patients could not walk by themselves after hip fracture surgery and it was performed by 3 experienced physical therapists using the predetermined protocols during the admission period. Physical therapy included physical modalities to reduce the postoperative pain, exercise to increase the range of motion and motor power of extremities, balance training, and gait training. Gait training was performed with a 4-legged front-rolling walker and progressed with a single cane. Single cane gait training started when the patients achieved his/her standing balance and weight shifting with a single cane for more than 10 minutes. If a patient achieved his/her balance and could walk in a stable manner with full weight bearing for more than 10 minutes, then gait training was continued without the use of an assistive device.

Pain killers, such as cetamadol, were temporarily used for pain control after surgery and their doses were adjusted according to the severity of the pain. Other medications for underlying diseases were continued during the admission period. Approximately 1 month after the surgery, patients were discharged to their home or other local hospitals for further care or management of medical problems.

\section{Data collection}

The demographic and baseline data of patients, including age, gender, presence of cognitive dysfunction, 
combined medical diseases, combined fracture, previous history of hip surgery, and prefracture ambulatory capacity, were collected from the medical charts. We defined the presence of cognitive dysfunction when we found the disease code for 'dementia' or 'cognitive dysfunction' in the medical charts or noticed that patients had been taking donepezil or memantine during the admission period. When the Mini-Mental State Examination score checked during the admission period was lower than 18 (moderate to severe cognitive dysfunction, as suggested by a previous article [14]), then we also considered this as the presence of 'cognitive dysfunction. When patients had medical diseases which could affect the ambulatory capacity, such as, stroke, Parkinsonism, deep vein thrombosis, and congestive heart failure, these patients were considered to have 'combined medical disease.' When the patients received surgery for other joints, such as wrist or knee joint or organs along with the hip joint, then they were considered to have 'combined fracture.'

Prefracture ambulatory capacity was classified into 'non-ambulatory status' (NA), 'ambulation with assistive device' (AA), 'independent-ambulation with single cane or without any assistive device' (IA). We classified ambulation with a single cane into the IA group, not the AA group, because many people over 60 years old walk with a single cane and they can step up and down and walk on the uneven ground with a single cane, even though they do not have any fracture.

These ambulatory capacities must be achieved without a helper, which is more than 5 in the Functional Independence Measure scoring criteria [15].

Data related to surgery was also gathered; days from the fracture to surgery, type of fracture, type of surgery, presence of postoperative complications, days from the surgery to physical therapy, and total admission period. The types of fracture were divided into femoral neck fracture (transcervical and subcapital bone fractures) and trochanteric fractures (intertrochanteric and subtrochanteric fractures). The types of surgery were also categorized into closed reduction and multiple pin insertion (CRP), open reduction and fixation with screw, intramedullary nail, or plate (ORF), and hemi-arthroplasty or total hip replacement arthroplasty (HRA). Infection, including aspiration pneumonia, postoperative delirium, skin problems including pressure sores, and other serious diseases caused by the surgery, were considered as postoperative complications. Physical therapies were carried out by 3 physical therapists. All of them had more than 10-years experience and they shared the same therapy protocol, so we did not think that the difference of therapy effect from each therapist was significant.

All medical records were reviewed with the permission of patients, and this study was approved by the Institutional Review Board of Samsung Medical Center.

\section{Outcome measures}

The postoperative ambulatory capacity of patients was investigated at approximately 1 month after hip surgery through an inpatient or outpatient medical chart, and it was classified into three categories, such as prefracture ambulatory capacity (NA, AA, and SA). We considered the assessment point as 1 month after hip surgery because 1 month was the period for discharge to home from the hospital or to complete the outpatient-based physical therapies.

\section{Statistical analysis}

Statistical analysis was performed with SAS software ver. 9.4 (SAS Institute, Cary, NC, USA). Continuous variables, including age, days from the fracture to surgery, days from the surgery to physical therapy, and total admission period (days) were expressed as the mean \pm standard deviation. Categorical variables, including the presence of cognitive dysfunction, combined medical diseases, combined fracture, and previous history of hip surgery were expressed as number of patients.

Using these variables, we set the prediction model through the multiple logistic regression analysis. To validate the prediction model, patients of more than 60 years old, who were admitted for hip fracture, underwent hip fracture surgery, received physical therapies, and had medical records about ambulatory capacities before falling and 1 month after surgery from January 2014 to December 2014, were selected as a validation set.

\section{RESULTS}

Among 548 patients, 147 patients were men and 401 patients were women and their mean age was $78.1 \pm 7.9$ years. Twenty-nine patients could not walk and were confined to a wheelchair before the fracture. Fifty-eight patients walked using their walkers, 143 patients walked 
using their single canes, and 318 patients walked without using any device before hip fracture. All baseline data are presented in Table 1.

Approximately 1 month after hip surgery, 166 patients could not walk (NA group), 188 patients walked with their assistive device (AA group), and 194 patients walked with single cane or without any assistive device (IA group). The average age of patients in the NA, AA, and IA groups was $79.5 \pm 7.4$ years, $79.2 \pm 7.8$ years, and $75.8 \pm 8.1$ years, respectively. The distribution of age, total admission period, days from the fracture to surgery, and days from the surgery to physical therapy according to the postoperative ambulatory capacity is presented in Fig. 1.

A score test for the proportional odds assumption showed that the chi-square value was 9.997 and its $p$ value was 0.002 , which did not meet the proportional odds assumption. Therefore, we had to use the partial proportional odds ratio and categorize the postoperative ambulatory capacity into NA plus AA and IA or NA and AA plus IA. The odds ratio of SA referenced by NA plus AA to age was $0.94(p<0.001)$, which means that if the age increases by one year, then the probability of IA will be 0.94 times compared to that of AA or NA. The odds ratio of IA plus AA, referenced by NA to age, was $0.97(\mathrm{p}=0.006)$, which means that if the age increases by 1 year, then the probability of IA or AA will be 0.97 times compared to that of NA (Table 1).

Major postoperative complications were infection, including aspiration pneumonia and urinary tract infection $(\mathrm{n}=15)$, postoperative delirium $(\mathrm{n}=14)$, skin problems including pressure sores and wound site oozing $(n=4)$, azotemia $(n=3)$, dyspnea including atelectasis $(n=3)$, cardiac arrhythmia $(n=1)$, stroke $(n=1)$, ileus $(n=1)$, and gall bladder perforation $(n=1)$.

Univariate analysis revealed that age, gender, total admission period, type of fracture, days from the fracture to

Table 1. Univariate analysis for postoperative ambulatory capacity after hip surgery

\begin{tabular}{|c|c|c|c|c|c|}
\hline & \multirow{3}{*}{$\begin{array}{c}\text { Total group } \\
(\mathbf{n}=\mathbf{5 4 8})\end{array}$} & \multicolumn{4}{|c|}{ Postoperative ambulatory capacity ${ }^{\text {a) }}$} \\
\hline & & \multicolumn{2}{|c|}{ Results for IA } & \multicolumn{2}{|c|}{ Results for IA plus AA } \\
\hline & & OR (95\% CI) & p-value & OR (95\% CI) & p-value \\
\hline Age (yr) & $78.1 \pm 7.9$ & $0.94(0.92-0.97)$ & $<0.001$ & $0.97(0.95-0.99)$ & 0.006 \\
\hline Gender, male & $147(26.82)$ & $1.74(1.18-2.56)$ & 0.005 & $1.07(0.71-1.62)$ & 0.748 \\
\hline Total admission period (day) & $23.9 \pm 18.0$ & $0.99(0.98-1.00)$ & $0.037^{*}$ & $0.99(0.98-1.00)$ & $0.037^{*}$ \\
\hline Type of fracture, femur neck & $101(18.43)$ & $1.43(1.25-1.61)$ & $0.036^{*}$ & $1.43(1.25-1.61)$ & $0.036^{*}$ \\
\hline Day to the surgery & $4.8 \pm 7.1$ & $0.97(0.95-0.99)$ & $0.007^{*}$ & $0.97(0.95-0.99)$ & $0.007^{*}$ \\
\hline \multicolumn{6}{|l|}{ Type of surgery } \\
\hline HRA & $252(45.7)$ & $0.417(0.97-1.02)$ & 0.059 & $0.791(0.82-1.43)$ & 0.760 \\
\hline ORF & $267(48.4)$ & $0.951(0.84-3.23)$ & 0.787 & $0.834(0.92-1.37)$ & 0.600 \\
\hline Day to the physical therapy & $6.5 \pm 4.7$ & $0.97(0.93-1.00)$ & $0.037^{*}$ & $0.97(0.93-1.00)$ & $0.037^{*}$ \\
\hline \multicolumn{6}{|l|}{ Prefracture ambulatory capacity ${ }^{\text {a) }}$} \\
\hline IA & $461(84.12)$ & $19.81(2.67-146.83)$ & 0.003 & $18.59(6.34-54.53)$ & $<0.001$ \\
\hline AA & $58(10.58)$ & $1.00(0.09-11.51)$ & 1.000 & $8.25(2.54-26.75)$ & $<0.001$ \\
\hline Combined medical diseases, present & $247(45.07)$ & $2.06(1.51-2.83)$ & $<0.001^{*}$ & $2.06(1.51-2.83)$ & $<0.001^{*}$ \\
\hline Combined fracture, present & $6(1.09)$ & $1.59(0.36-7.02)$ & $0.543^{*}$ & $1.59(0.36-7.02)$ & $0.543^{*}$ \\
\hline Postoperative complication, present & $43(7.85)$ & $8.11(2.48-26.58)$ & 0.001 & $2.14(1.14-4.01)$ & 0.018 \\
\hline Cognitive dysfunction, present & $68(12.41)$ & $4.75(2.22-10.15)$ & $<0.001$ & $2.141(1.28-3.59)$ & 0.004 \\
\hline
\end{tabular}

Values are presented as mean \pm standard deviation or the number of patients (\%).

IA, independent-ambulation without device; AA, ambulation with assistive device; HRA, hemi-arthroplasty or total hip replacement arthroplasty; ORF, open reduction and fixation with screw, intramedullary nail, or plate; OR, odds ratio; CI, confidence interval.

${ }^{a)}$ Ambulatory capacity was divided into three categories of non-ambulatory status, ambulation with assistive device, and independent-ambulation without device.

*Proportional odds model for results of postoperative ambulatory capacity. 
(A)

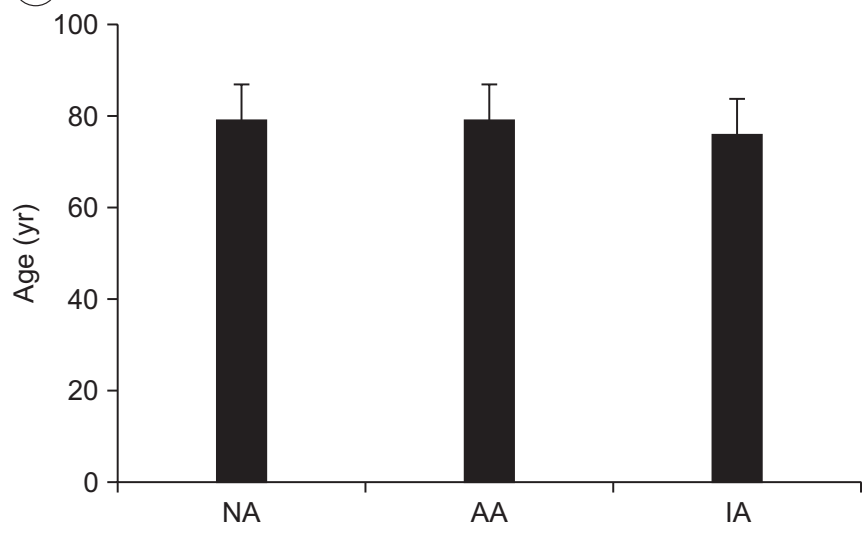

(C)

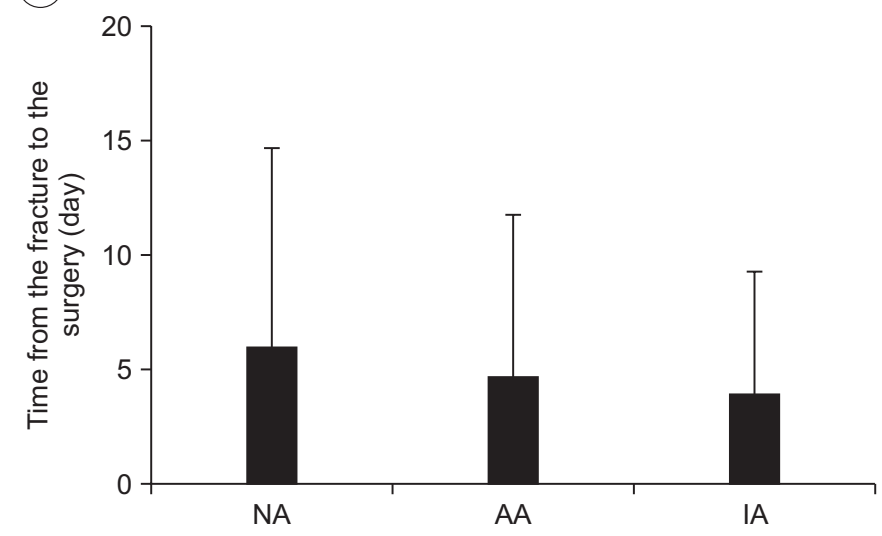

(B)

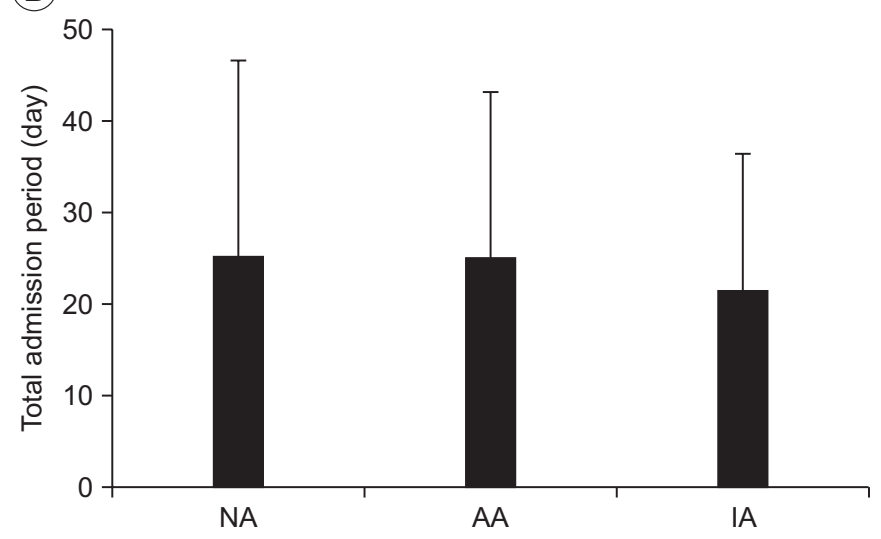

(D)

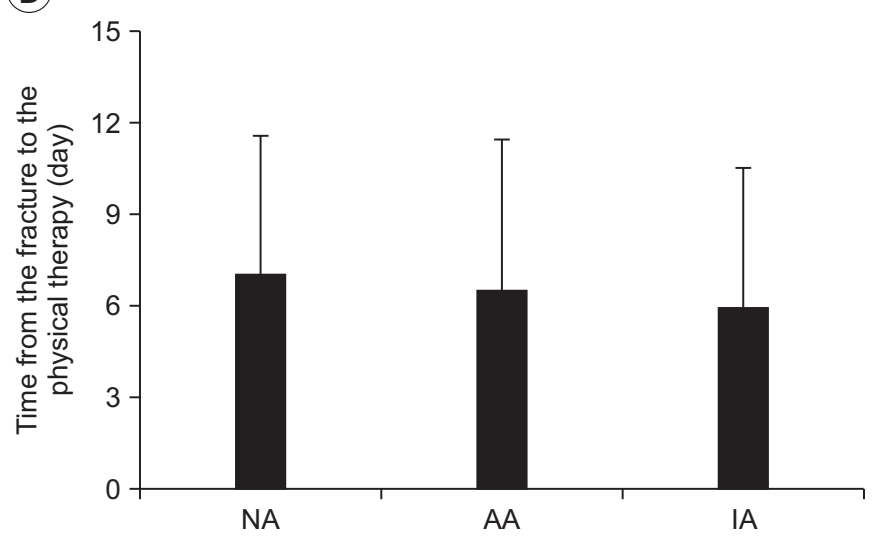

Fig. 1. (A) Distribution of age, (B) total admission period, (C) days from the fracture to surgery, and (D) days from the surgery to rehabilitation, according to the postoperative ambulatory capacity presented. The boxes are averages and the lines are one standard deviation. NA, non-ambulatory status; AA, ambulation with assistive device; IA, independent-ambulation without device.

Table 2. Multiple-variable analysis of postoperative ambulatory capacity after hip surgery

\begin{tabular}{|c|c|c|c|c|}
\hline & \multicolumn{4}{|c|}{ Postoperative capacity } \\
\hline & \multicolumn{2}{|l|}{ IA } & \multicolumn{2}{|c|}{ IA plus AA } \\
\hline & OR (95\% CI) & p-value & OR (95\% CI) & p-value \\
\hline Age (yr) & $0.94(0.92-0.97)$ & $<0.001$ & $0.96(0.93-0.98)$ & 0.001 \\
\hline Gender, male & $1.64(1.08-2.51)$ & 0.021 & $0.98(0.63-1.55)$ & 0.943 \\
\hline \multicolumn{5}{|l|}{ Prefracture ambulatory capacity ${ }^{a}$} \\
\hline IA & $19.17(2.59-141.85)$ & 0.004 & $16.72(5.64-49.6)$ & $<0.001$ \\
\hline AA & $1.26(0.11-14.47)$ & 0.854 & $9.46(2.88-31.11)$ & $<0.001$ \\
\hline Combined medical diseases, absent & $2.02(1.44-2.85)$ & $<0.001^{*}$ & $2.02(1.44-2.85)$ & $<0.001^{*}$ \\
\hline
\end{tabular}

IA, independent-ambulation without device; AA, ambulation with assistive device; OR, odds ratio; CI, confidence interval.

${ }^{a)}$ Ambulatory capacity was divided into three categories of non-ambulatory status, ambulation with assistive device, and self-ambulation without device.

*Proportional odds model for results of postoperative ambulatory capacity. 
surgery, days from the surgery to physical therapy, prefracture ambulatory capacity, combined medical disease, postoperative complications, and cognitive dysfunction were significantly related to the postoperative ambulatory capacity. All odds ratios are presented in Table 1. Multiple-variable analysis was performed using these factors for the prediction of postoperative ambulatory capacity and among them, age, gender, prefracture ambulatory capacity, and combined medical disease were found to be the factors related to postoperative ambulatory capacity (Table 2). Proportions of postoperative ambulatory capacity according to the prefracture ambulatory capacity, gender, and presence of combined medical disease are presented in Fig. 2. Using these four related factors, the prediction model was established.

The equation of the prediction model is presented in Fig. 3.

For example, if an 80-year-old woman who walks without any assistive device and has no medical disease falls down and receives hip surgery, she will walk with a single cane or without any assistive device after physical ther-

(A)

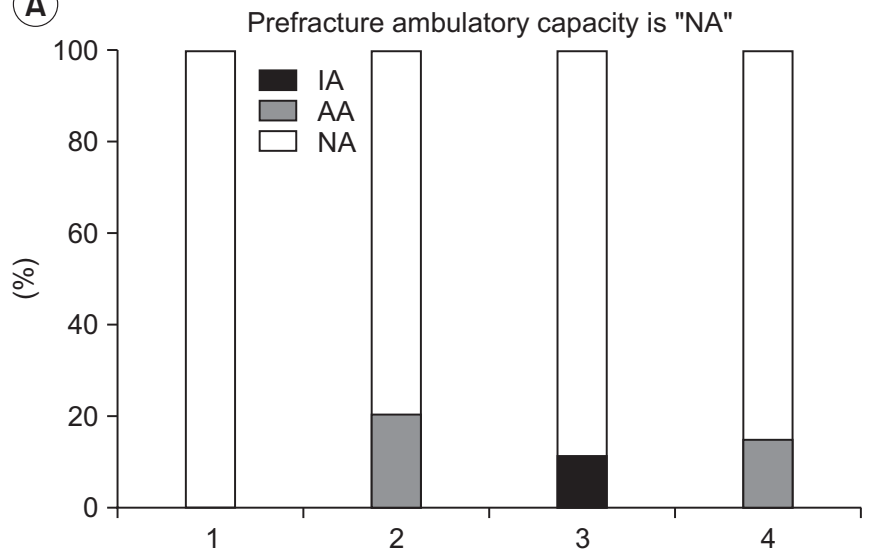

(C)

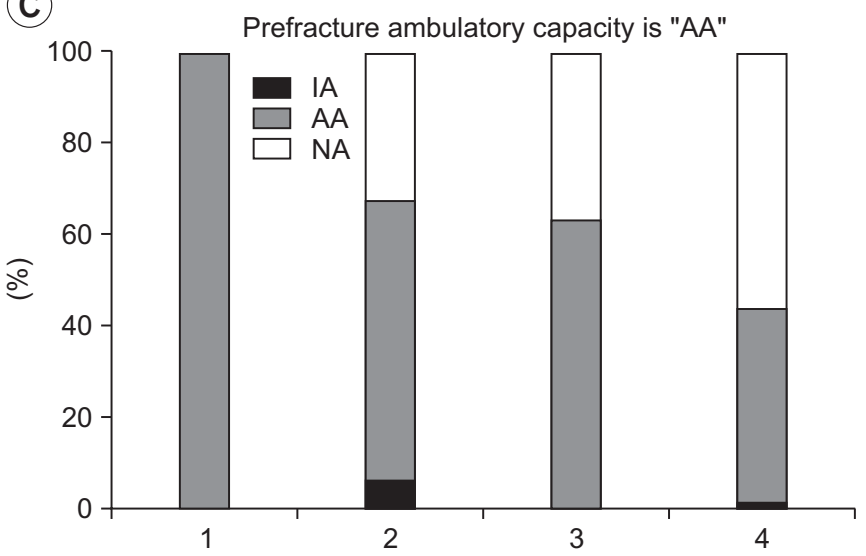

apy at postoperative one month with $41.5 \%$ probability, she will walk with assistive device with $37.8 \%$ probability, and she will not walk with $20.7 \%$ probability. If instead, a man has the same condition, the probability of walk-

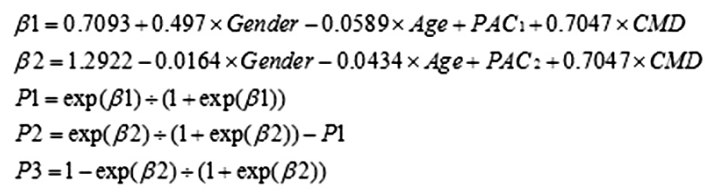

P1: chance of postoperative ambulatory capacity to be 'SA'

P2: chance of postoperative ambulatory capacity to be ' $A A$ '

P3: chance of postoperative ambulatory capacity to be 'NA'

Fig. 3. The equation of the prediction model. NA, nonambulatory status; AA, ambulation with assistive device; IA, independent-ambulation without device.

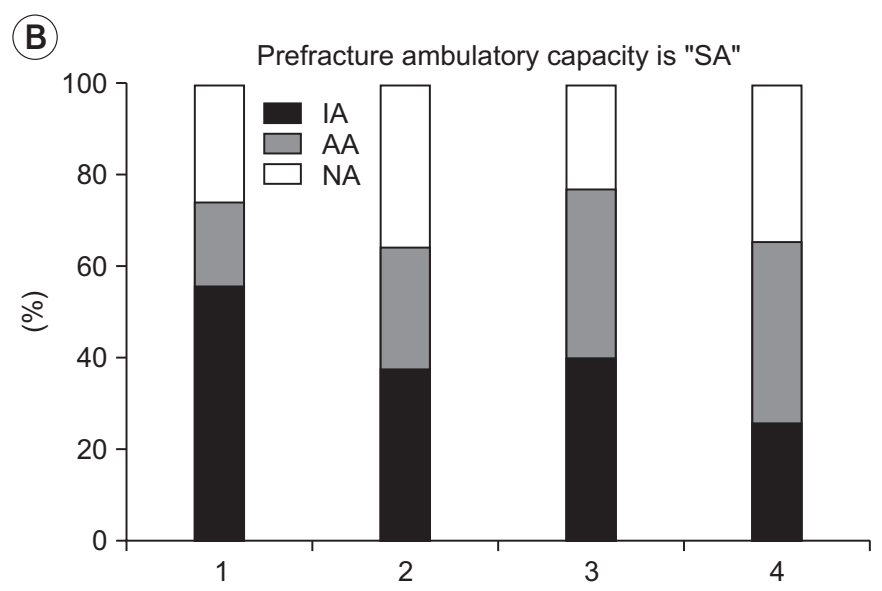

Fig. 2. Proportions of postoperative ambulatory capacity are presented according to the prefracture ambulatory capacity, gender, and presence of combined medical disease. (A) Prefracture ambulatory capacity is "NA", (B) prefracture ambulatory capacity is "IA", (C) prefracture ambulatory capacity is "AA". NA, non-ambulatory status; AA, ambulation with assistive device; IA, independentambulation without device; 1, Man, no combined medical disease; 2, Man, combined medical disease; 3, Woman, no combined medical disease; 4 , Woman, combined medical disease. 


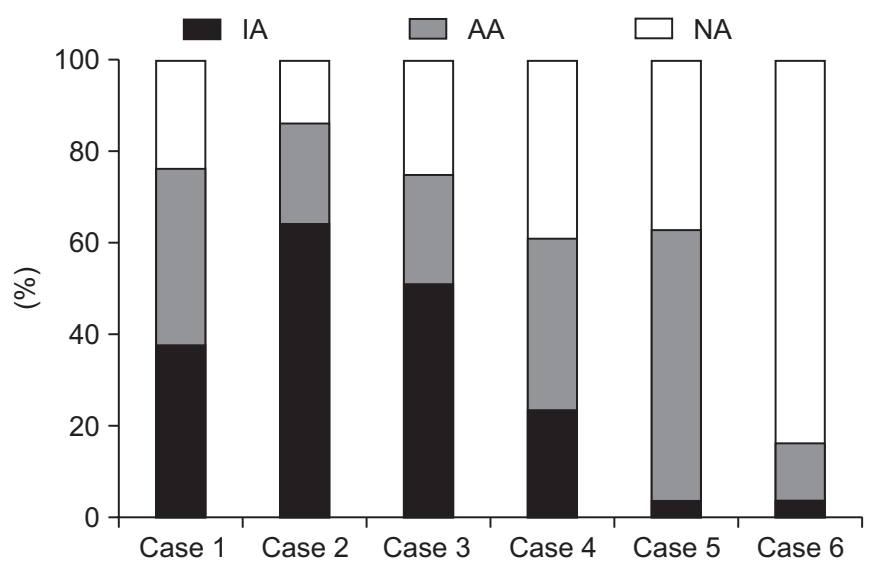

Fig. 4. Probability of postoperative ambulatory status in several cases. IA, independent-ambulation without device, AA, ambulation with assistive device; NA, nonambulatory status.

ing with a single cane or without any assistive device will increase to $53.8 \%$. If this patient is assumed to have combined medical disease along with the same condition, the probability of walking with single cane or without any assistive device will decrease to $25.9 \%$. If the age decreases to 60 years and the other conditions are the same, the probability of walking with a single cane or without any assistive device will increase to $69.7 \%$. The hypothetical cases that show the probability of postoperative ambulatory capacity are presented in Fig. 4. Table 3 shows details of each hypothetical case. The generalized $R^{2}$ of this prediction model was 0.240 and the accuracy of this prediction model was 0.704 when applied to the validation set.

\section{DISCUSSION}

In our study, we found that age, gender, prefracture ambulatory capacity, and combined medical diseases were the factors related to the postoperative ambulatory capacity. Using these factors, we built the prediction model of postoperative ambulatory capacity after physical therapy following hip fracture surgery.

Although the World Health Organization accepts the chronological age of 65 years as a definition of 'elderly' person, we have included patients more than 60 years old. From our hospital data, most hip fracture surgeries were conducted in people of more than 60 years old, so we included patients of more than 60 years old.

Results of our study were consistent with those of previous studies which demonstrated that age, gender, and
Table 3. Characteristics of the hypothetical cases

\begin{tabular}{llllc}
\hline & $\begin{array}{c}\text { Age } \\
\text { (yr) }\end{array}$ & Gender & $\begin{array}{c}\text { Combined } \\
\text { disease }\end{array}$ & $\begin{array}{c}\text { Prefracture } \\
\text { ambulatory } \\
\text { capacity }\end{array}$ \\
\hline Case 1 & 80 & Woman & Absence & IA \\
Case 2 & 60 & Woman & Absence & IA \\
Case 3 & 80 & Man & Absence & IA \\
Case 4 & 80 & Woman & Presence & IA \\
Case 5 & 80 & Woman & Absence & AA \\
Case 6 & 80 & Woman & Absence & NA \\
\hline
\end{tabular}

IA, independent-ambulation without device; AA, ambulation with assistive device; NA, non-ambulatory status.

prefracture ambulatory capacity were significant risk factors that predict the functional improvement $[16,17]$. Holt et al. [16] and Lieberman et al. [18] already proved that advanced age and male gender were the known risk factors of mortality and functional deterioration after hip fracture. The major difference between our study and their studies was that we divided the ambulatory capacity of patients into ambulation with a device and ambulation without a device and we found the factors related to movement of each step (from NA to AA and from AA to IA). Above all, we set up the prediction model through the partial proportional odds ratios, and this was the first study to do so.

Some factors were significantly related to movement from one step to another step, but they were not significantly related to movement from another step to the other step.

For example, gender was a significant factor that can predict whether patients can walk without a device or not (odds ratio $=1.74, p=0.005$ ), but this was not the case when predicting whether patients can walk or cannot walk (odds ratio $=1.07, \mathrm{p}=0.748$ ). Therefore, gender was a significant factor only when determining whether a patient can walk without any device or not, but not in determining whether a patient can walk with a device or cannot walk. Considering that women were more likely to be unable to hold a tandem stand than men, as observed in the study by Keevil et al. [19], it is plausible that gender is an important risk factor for deciding the need for an ambulatory device.

Total admission periods, days from the fracture to surgery, days from the surgery to physical therapy, postoperative complications, and cognitive dysfunction were 
significant risk factors that affected the ambulation after the surgery irrespective of need for an ambulatory device in the univariate analysis. However, these relations disappeared in the multivariate analysis, which might be due to the fact that age, gender, and prefracture ambulatory capacity affected these factors.

Type of fracture was found to be related to the postoperative ambulatory capacity, consistent with other studies $[17,20]$. Our study showed a higher incidence of intertrochanteric and subtrochanteric fractures than that of femoral neck fracture. This might be due to the fact that patients with femoral neck fracture showed better function than those with intertrochanteric and subtrochanteric fracture [20]; therefore, a higher number of patients with femoral neck fracture were discharged from hospital without physical therapy, and were excluded from our study.

Mathew et al. [21] and Kenzora et al. [22] demonstrated that the presence of combined medical disease, especially stroke and congestive heart failure was related to increased mortality or poor functional outcome in many studies. This finding was consistent with that in our study, and we also demonstrated that combined medical disease can affect the need for an assistive device after hip fracture surgery, in addition to the possibility of ambulation.

We investigated patient ambulatory capacity at approximately one month after the surgery, at which point most patients had been discharged from our hospital or had completed the outpatient-based physical therapies. This study focused on ambulatory capacity after hip fracture surgery and physical therapies in patients with hip fracture, and it did not focus on the occurrence of refracture or other serious medical events, which could affect the ambulatory capacity but did not have a relation to the hip fracture. We thought that a 1-month time period was sufficient for the recovery of the patients' maximal ambulatory capacity, and according to further follow-up data from some patients, most of their postoperative ambulatory capacity did not change if they did not suffer from any other disease.

There are several limitations to this study. We identified that age, gender, prefracture ambulatory capacity, and combined medical disease are associated with the postoperative ambulatory capacity; whether a patient can walk without a device or not, or whether a patient can walk or cannot walk. Unfortunately, all of these factors cannot be corrected after occurrence of the event. For the limitation of a retrospective study design, the accuracy of this prediction model was relatively low. Further prospective studies should be constructed to identify the correctable factors related to the improvement of the postoperative ambulatory capacity and to increase the accuracy of the prediction model.

Using this model, we can predict the ambulatory capacity following hip fracture surgery. Further prospective studies should be constructed to improve the postoperative ambulatory capacity, following hip fracture surgery.

\section{CONFLICT OF INTEREST}

No potential conflict of interest relevant to this article was reported.

\section{ACKNOWLEDGMENTS}

This research was supported by the Basic Science Research Program through the National Research Foundation of Korea (NRF) funded by the Ministry of Education, Science and Technology (No. 2013R1AlA1007404).

\section{REFERENCES}

1. Fuller GF. Falls in the elderly. Am Fam Physician 2000;61:2159-68, 2173-4.

2. Rose S, Maffulli N. Hip fractures: an epidemiological review. Bull Hosp Jt Dis 1999;58:197-201.

3. Lim JY, Park WB, Oh MK, Kang EK, Paik NJ. Falls in a proportional region population in Korean elderly: incidence, consequences, and risk factors. J Korean Geriatr Soc 2010;14:8-17.

4. Cooper C. The crippling consequences of fractures and their impact on quality of life. Am J Med 1997;103: 12S-19S.

5. Dubljanin-Raspopovic E, Markovic Denic L, Marinkovic J, Grajic M, Tomanovic Vujadinovic S, Bumbasirevic M. Use of early indicators in rehabilitation process to predict one-year mortality in elderly hip fracture patients. Hip Int 2012;22:661-7.

6. Van Hook FW, Demonbreun D, Weiss BD. Ambulatory devices for chronic gait disorders in the elderly. Am Fam Physician 2003;67:1717-24. 
7. Levin P. Ambulatory assistive devices in orthopaedics: uses and modifications. J Am Acad Orthop Surg 2010;18:315-6.

8. Kristensen MT. Factors affecting functional prognosis of patients with hip fracture. Eur J Phys Rehabil Med 2011;47:257-64.

9. Hershkovitz A, Kalandariov Z, Hermush V, Weiss R, Brill S. Factors affecting short-term rehabilitation outcomes of disabled elderly patients with proximal hip fracture. Arch Phys Med Rehabil 2007;88:916-21.

10. Folden S, Tappen R. Factors influencing function and recovery following hip repair surgery. Orthop Nurs 2007;26:234-41.

11. Foss NB, Kristensen MT, Kehlet H. Prediction of postoperative morbidity, mortality and rehabilitation in hip fracture patients: the cumulated ambulation score. Clin Rehabil 2006;20:701-8.

12. Semel J, Gray JM, Ahn HJ, Nasr H, Chen JJ. Predictors of outcome following hip fracture rehabilitation. PM R 2010;2:799-805.

13. Bellelli G, Noale M, Guerini F, Turco R, Maggi S, Crepaldi G, et al. A prognostic model predicting recovery of walking independence of elderly patients after hipfracture surgery: an experiment in a rehabilitation unit in Northern Italy. Osteoporos Int 2012;23:2189200.

14. Mungas D. In-office mental status testing: a practical guide. Geriatrics 1991;46:54-8, 63, 66.

15. Bohannon RW. Scoring transfer and locomotion independence of home care patients: Barthel versus functional independence measure. Int J Rehabil Res
1999;22:65-6.

16. Holt G, Smith R, Duncan K, Hutchison JD, Gregori A. Gender differences in epidemiology and outcome after hip fracture: evidence from the Scottish Hip Fracture Audit. J Bone Joint Surg Br 2008;90:480-3.

17. Lee D, Jo JY, Jung JS, Kim SJ. Prognostic factors predicting early recovery of pre-fracture functional mobility in elderly patients with hip fracture. Ann Rehabil Med 2014;38:827-35.

18. Lieberman D, Fried V, Castel H, Weitzmann S, Lowenthal MN, Galinsky D. Factors related to successful rehabilitation after hip fracture: a case-control study. Disabil Rehabil 1996;18:224-30.

19. Keevil VL, Hayat S, Dalzell N, Moore S, Bhaniani A, Luben $\mathrm{R}$, et al. The physical capability of communitybased men and women from a British cohort: the European Prospective Investigation into Cancer (EPIC)Norfolk study. BMC Geriatr 2013;13:93.

20. Haentjens P, Autier P, Barette M, Venken K, Vanderschueren D, Boonen S, et al. Survival and functional outcome according to hip fracture type: a one-year prospective cohort study in elderly women with an intertrochanteric or femoral neck fracture. Bone 2007;41:958-64.

21. Mathew RO, Hsu WH, Young Y. Effect of comorbidity on functional recovery after hip fracture in the elderly. Am J Phys Med Rehabil 2013;92:686-96.

22. Kenzora JE, McCarthy RE, Lowell JD, Sledge CB. Hip fracture mortality: relation to age, treatment, preoperative illness, time of surgery, and complications. Clin Orthop Relat Res 1984;(186):45-56. 